\title{
89Zr-nimotuzumab for immunoPET imaging of epidermal growth factor receptor I
}

\author{
Rufael Chekol ${ }^{1,2}$, Viswas Raja Solomon ${ }^{1,2}$, Elahe Alizadeh ${ }^{1,2}$, Wendy Bernhard ${ }^{3}$, \\ Darrell Fisher ${ }^{4}$, Wayne Hill ${ }^{3}$, Kris Barreto ${ }^{3}$, John Francis DeCoteau ${ }^{3}$, Angel Casaco \\ Parada $^{5}$, Clarence Ronald Geyer ${ }^{3}$ and Humphrey Fonge ${ }^{1,2,6}$ \\ ${ }^{1}$ Department of Medical Imaging, University of Saskatchewan, College of Medicine, Saskatoon, SK, Canada \\ ${ }^{2}$ Saskatchewan Centre for Cyclotron Sciences (SCCS), The Fedoruk Centre, Saskatoon, SK, Canada \\ ${ }^{3}$ Department of Pathology and Laboratory Medicine, University of Saskatchewan, College of Medicine, Saskatoon, SK, Canada \\ ${ }^{4}$ Versant Medical Physics and Radiation Safety, Boston, MA, USA \\ ${ }^{5}$ Centre for Molecular Immunology, Havana, Cuba \\ ${ }^{6}$ Department of Medical Imaging, Royal University Hospital Saskatoon, Saskatoon, SK, Canada \\ Correspondence to: Humphrey Fonge, email: humphrey.fonge@usask.ca \\ Clarence Ronald Geyer, email: ron.geyer@usask.ca
}

Keywords: epidermal growth factor receptor l; zirconium-89; nimotuzumab; immunoPET/CT; radiochemistry

Received: January 08, $2018 \quad$ Accepted: February 26, $2018 \quad$ Published: March 30, 2018

Copyright: Chekol et al. This is an open-access article distributed under the terms of the Creative Commons Attribution License 3.0 (CC BY 3.0), which permits unrestricted use, distribution, and reproduction in any medium, provided the original author and source are credited.

\section{ABSTRACT}

Rationale: Epidermal growth factor receptor (EGFR) upregulation is associated with enhanced proliferation and drug resistance in a number of cancers. Nimotuzumab is a humanized monoclonal antibody with high affinity for EGFR. The objective of this study was to determine if ${ }^{89} \mathrm{Zr}$-DFO-nimotuzumab could be suitable for human use as a PET probe for quantifying EGFR in vivo.

Methods: To evaluate the pharmacokinetics, biodistribution, microPET imaging, radiation dosimetry, and normal tissue toxicity in tumor and non-tumor bearing mice of ${ }^{89} \mathrm{Zr}$-desferoxamine-nimotuzumab ( ${ }^{89} \mathrm{Zr}$-DFO-nimotuzumab) of a product prepared under GMP conditions. Nimotuzumab was conjugated to DFO and radiolabeled with ${ }^{89} \mathrm{Zr}$. ${ }^{89} \mathrm{Zr}$-DFO-nimotuzumab was characterized by in vitro gel-electrophoresis, biolayer interferometry (BLI) and flow cytometry. ${ }^{89} \mathrm{Zr}$-DFO-nimotuzumab was evaluated in vivo by microPET and ex vivo by biodistribution in healthy and EGFR-positive tumor bearing mice.

Results: Flow cytometry with A431 cells showed no significant difference in the dissociation constant of nimotuzumab (13 $\pm 2 \mathrm{nM})$ compared with DFO-nimotuzumab $(17 \pm 4 \mathrm{nM})$. PET imaging in mice xenografts showed persistently high tumor uptake with the highest uptake obtained in DLD-1 xenograft (18.3\%IA/cc) at $168 \mathrm{hp} . \mathrm{i}$. The projected human effective dose was low and was $0.184 \mathrm{mSv} / \mathrm{MBq}(0.679 \mathrm{rem} / \mathrm{mCi})$ in females and $0.205 \mathrm{mSv} / \mathrm{MBq}(0.757 \mathrm{rem} / \mathrm{mCi})$ in males. There was no apparent normal tissue toxicity as shown by cell blood counts and blood biochemistry analyses at 168-fold and 25-fold excess of the projected human radioactive and mass dose of the agent.

Conclusion: ${ }^{89} \mathrm{Zr}-\mathrm{DFO}-\mathrm{nimotuzumab}$ had low organ absorbed dose and effective dose that makes it suitable for potential human use. 


\section{INTRODUCTION}

Overexpression of EGFR is implicated in all aggressive cancers of epithelial origin including squamous cell head \& neck $(90-100 \%)$ [1], glioma $(90-100 \%)$ [2], non-small cell lung $(75-90 \%)$, colorectal $(80-85 \%)$ [3], breast $(20-30 \%)$ [4] and cervical [5] cancers. AntiEGFR antibodies e.g. cetuximab, panitumumab, and nimotuzumab have been used for treating different EGFRpositive cancers. With the exception of nimotuzumab, these antibodies cause significant cutaneous toxicity in $45-100 \%$ of patients. In contrast, nimotuzumab is better tolerated and has low skin toxicities, because its "affinity optimized" binding characteristics ensures transient binding to low EGFR-expressing healthy tissues such as the skin. Garrido et al [6] showed that the low skin toxicity of nimotuzumab is attributed to its transient monovalent binding in low-EGFR expressing tissues such as the skin and gastro-intestinal mucosa (these tissues account for the dose-limiting toxicities observed with anti-EGFR antibody treatments). This low transient monovalent binding is due to a 10-fold lower affinity of nimotuzumab for EGFR compared to cetuximab or panitumumab [6].

Existing ex vivo assays for monitoring EGFR expression such as immunohistochemistry (IHC), fluorescence in situ hybridization (FISH), and quantitative polymerase chain reaction (qPCR) are invasive and not very reliable $[7,8]$. These techniques require frequent biopsies that are impossible for most patients. In addition, biopsy sampling is inherently flawed because of the intra- and inter-lesion heterogeneity of tumors. It is also well known that the EGFR expression changes over time [9]. In vivo measurement of the EGFR expression in cancer will offer several advantages over current ex vivo methods, including measuring EGFR expression over the entire tumor volume rather than just a part of the tumor, assessing the biologic availability of EGFR in vivo, evaluating effects of therapy on EGFR expression, and quantifying EGFR expression of all lesions in real time.

A few ImmunoPET probes have been developed to image EGFR expression in vivo [10-14]. ${ }^{89} \mathrm{Zr}$-labeled desferoxamine (DFO) conjugated cetuximab ${ }^{89} \mathrm{Zr}$-DFOcetuximab) is currently been investigated in clinical trials for PET imaging of EGFR [11, 14]. Menke-van der Houven van Oordt et al. administered $37 \mathrm{MBq}$ of ${ }^{89} \mathrm{Zr}$ DFO-cetuximab to 10 colorectal cancer patients with wild-type KRAS mutations two hours after treatment with therapeutic doses of cetuximab. PET scans were acquired at 1 to 10 days post ${ }^{89} \mathrm{Zr}$-DFO-cetuximab injection [14]. $6 / 10$ patients that had positive ${ }^{89} \mathrm{Zr}$ lesions showed clinical benefit with cetuximab, while $4 / 10$ patients with no positive ${ }^{89} \mathrm{Zr}$-DFO-cetuximab lesions detected by PET did not show clinical response to cetuximab. The strong positive correlation between ${ }^{89} \mathrm{Zr}$-DFO-cetuximab uptake and clinical response to cetuximab treatment implies that this imaging agent can be used to select patients that would benefit from cetuximab therapy. Because of its low binding to tissues that express low EGFR, such as skin, a nimotuzumab immunoPET agent may be potentially advantageous over ${ }^{89} \mathrm{Zr}$-DFO-cetuximab or ${ }^{89} \mathrm{Zr}$-DFOpanitumumab probes as the low off target binding may provide a more favorable dosimetry and unequivocal delineation of secondary lesions in organs such as the liver that express low EGFR and are frequent metastatic sites for many EGFR positive cancers. Such a favorable dosimetry may also permit repeated tracer injection.

A few probes have been developed using nimotuzumab for imaging EGFR expression by SPECT. Vallis et al. evaluated a ${ }^{99 \mathrm{~m}} \mathrm{Tc}$-nimotuzumab in a phase I trial [15]. In this study 12 patients received $999 \mathrm{MBq}$ of ${ }^{99 \mathrm{~m}} \mathrm{Tc}$-nimotuzumab and were imaged at $30 \mathrm{~min}$ for up to 24 $\mathrm{h}$ post injection. The effective dose of ${ }^{99 \mathrm{~m}} \mathrm{Tc}$-nimotuzumab was $1.34 \pm 0.02 \times 10^{-8} \mathrm{mSv} \mathrm{Bq}^{-1}$. One patient with squamous cell carcinoma of the mouth showed a positive scan. EGFR positivity was not an entry criteria for this trial. PET offers unique advantages such as superior spatial resolution, better signal to-noise ratio and quantitative capabilities over SPECT which makes it the modality of choice for radiolabeled antibodies/fragment. Our goal was to develop a clinical-grade ${ }^{89} \mathrm{Zr}$-DFO-nimotuzumab for imaging EGFR expression using PET. Here, we describe the development and, in vitro and in vivo characterization of ${ }^{89} \mathrm{Zr}$-DFO-nimotuzumab that will meet all Health Canada guidelines for a phase I clinical study.

\section{RESULTS}

\section{Conjugation and quality control of DFO- nimotuzumab kit formulation}

The conjugation of $p$-Bz-SCN-DFO to nimotuzumab resulted in an average of two DFO molecules per antibody molecule as determined by the difference in molecular weight of nimotuzumab and DFO-nimotuzumab as measured by bioanalyzer. Nimotuzumab and DFO-nimotuzumab were $>95 \%$ pure on HPLC with $<2 \%$ aggregates in the latter. Following conjugation, the resulting DFO-nimotuzumab kit was characterized for stability, binding to recombinant EGFR, aggregation, and size. In stability studies, samples that were stored at $4^{\circ} \mathrm{C}$ showed $<4 \%$ degradation while no degradation was seen with samples stored at $-80^{\circ} \mathrm{C}$ for over 6 months. Bioanalyzer confirms that nimotuzumab and DFO-nimotuzumab were $>95 \%$ pure with molecular weights of $161.5 \mathrm{kDa}$ and $162.7 \pm 0.9 \mathrm{kDa}$, respectively (Supplementary Figure 1A-1B). The conjugation of DFO to nimotuzumab had a modest but significant impact on the binding to recombinant EGFR by BLI (Figure 1A). The $K_{D}$ was significantly different $(p<0.0001)$ between nimotuzumab $(23 \pm 3 \mathrm{nM})$ and DFO-nimotuzumab $(30 \pm 1$ $\mathrm{nM})$. There was no difference in the association rate $\left(\mathrm{k}_{\mathrm{on}}\right)$, however, there was a 1.3 -fold increase $(p<0.0001)$ in the 


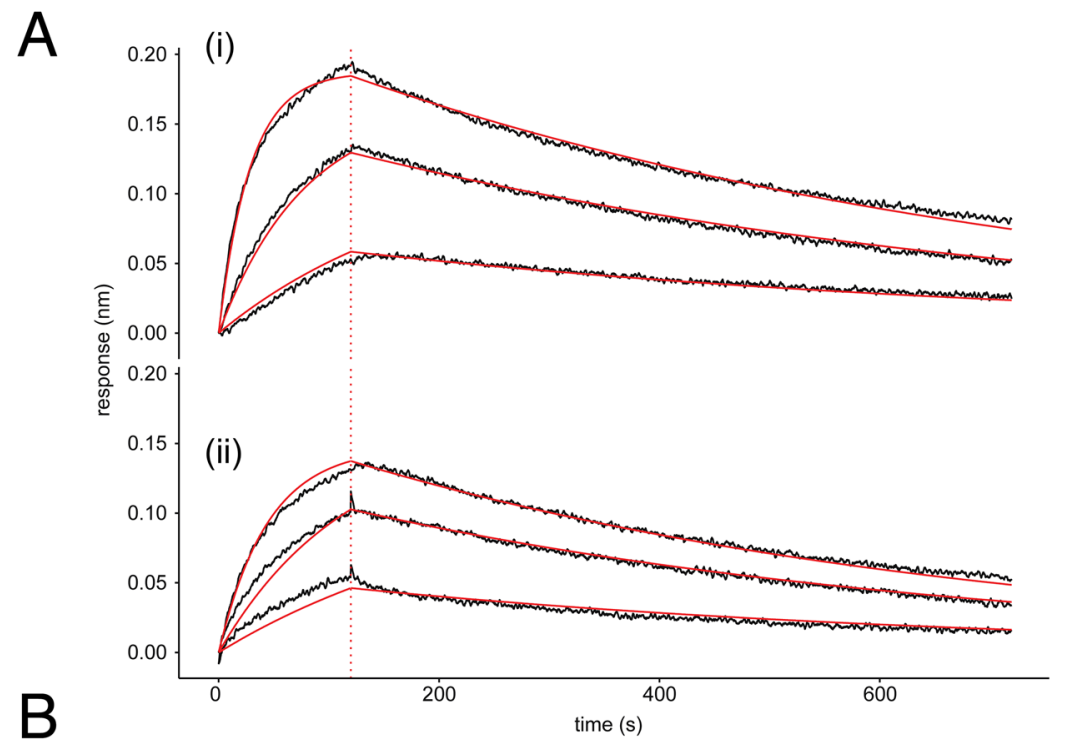

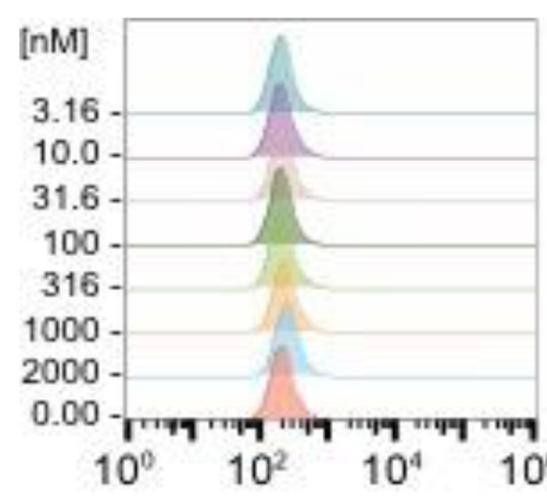

(i)

C

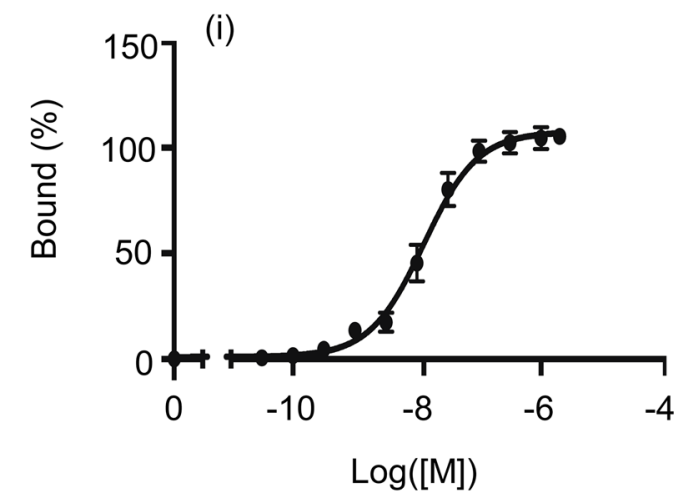

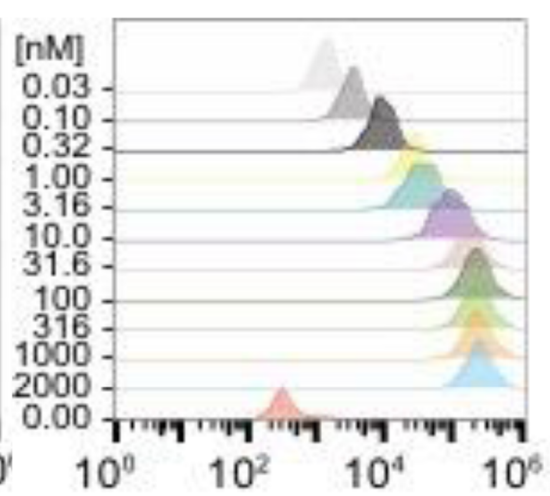

(ii)

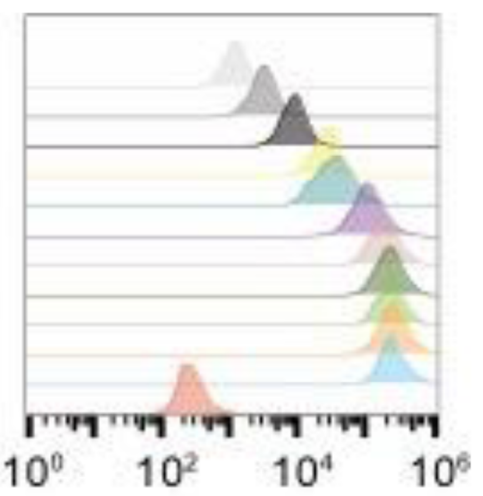

(iii)

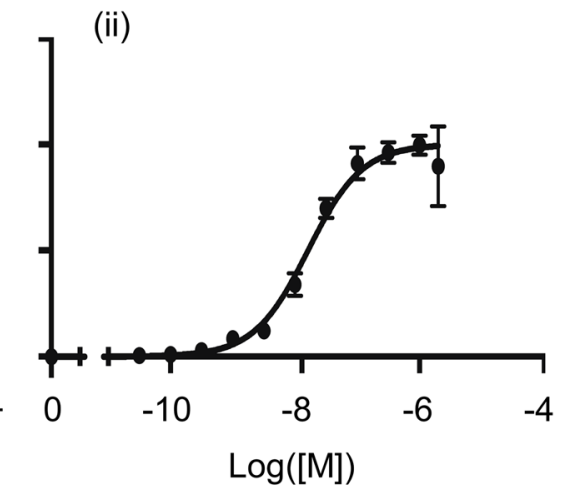

Figure 1: (A-C) In vitro characterization of DFO-nimotuzumab kit formulation by biolayer interferometry (BLI) and flow cytometry. (A) BLI binding curves for nimotuzumab (i) and DFO-nimotuzumab (ii) at different concentrations (55.5, 166.7 and $500 \mathrm{nM}$ ). A $\mathrm{K}_{\mathrm{D}}$ of $23 \pm 3 \mathrm{nM}$ ) and $48 \pm 9 \mathrm{nM}$ was obtained for nimotuzumab and DFO-nimotuzumab, respectively. Solid black line indicates raw data, solid red line indicates curve fit, vertical dashed red line indicates transition from association to dissociation phase. (B) Flow cytometry results of nimotuzumab in control MDA-MB-435 (i) with very low levels of EGFR and corresponding EGFR-positive A431 cells (ii and iii). No substantial binding was seen in MDA-MB-435 cells. Binding of DFO-nimotuzumab (iii) in A431 cells was comparable with nimotuzumab (ii). (C) Nimotuzumab (i) and DFO-nimotuzumab (ii) showed saturation binding by flow cytometry with a $\mathrm{K}_{\mathrm{D}}$ of $13 \pm 2 \mathrm{nM}$ and $17 \pm 4 \mathrm{nM}$, respectively. 
dissociation rate of DFO-nimotuzumab $\left(2.0 \times 10^{-3} \pm 0.8\right.$ $\left.\times 10^{-4} \mathrm{~s}-1\right)$ compared with nimotuzumab $\left(1.5 \times 10^{-3} \pm 2\right.$ $\left.\times 10^{-4} \mathrm{~s}-1\right)$. In vitro binding of nimotuzumab and DFOnimotuzumab to high EGFR expressing A431 cell line was determined using flow cytometry. There was no significant difference in receptor binding of DFO-nimotuzumab $\left(\mathrm{K}_{\mathrm{D}}\right.$ $17 \pm 2 \mathrm{nM}$ : Figure 1B, iii and Figure 1C, ii) compared with nimotuzumab $\left(\mathrm{K}_{\mathrm{D}} 13 \pm 2 \mathrm{nM}\right.$ : Figure $1 \mathrm{~B}$, ii and Figure 1C, i). DFO-nimotuzumab did not bind control MDA-MB-435 cell line (Figure 1B, i). Stability data obtained by HPLC were identical to those obtained using the bioanalyzer (Supplementary Figure 1C). Similarly, stability studies showed no change in binding affinity by biolayer interferometry (BLI) over the studied period.

\section{Radiolabelling and characterization of ${ }^{89} \mathrm{Zr}$ - DFO-nimotuzumab}

The optimal condition to obtain quantitative labeling yield was $\mathrm{pH} 7 \pm 0.2$, temperature of $37^{\circ} \mathrm{C}$ and two $\mathrm{hr}$ incubation. To obtain quantitative radiolabeling yield without the need to purify before drug administration, a range of specific activities were tested. The labeling efficiency of ${ }^{89} \mathrm{Zr}$-DFO-nimotuzumab was $91 \pm 1.2 \%$ with a specific activity of $0.5 \mathrm{MBq} / \mu \mathrm{g}$ (Table 1). High radiochemical yield of $94 \pm 0.9 \%$ was obtained albeit at a lower specific activity $(0.1 \mathrm{MBq} / \mu \mathrm{g})$. A radiochemical purity of $>98 \%$ was obtained after purification. To investigate product shelf-life, ${ }^{89} \mathrm{Zr}$-DFO-nimotuzumab was analyzed at different time periods using HPLC, following storage at $4^{\circ} \mathrm{C}$ or room temperature (RT). Starting with $94 \%$ pure ${ }^{89} \mathrm{Zr}$-DFO-nimotuzumab, we obtained $>90 \%$ radiochemical purity after $24 \mathrm{~h}$ storage at room temperature (RT) (Supplementary Figure 2 and Supplementary Table 1). Loss of ${ }^{89} \mathrm{Zr}$ was slightly higher at RT than at $4^{\circ} \mathrm{C}$. A maximum shelf-life of $24 \mathrm{~h}$ is recommended at $4^{\circ} \mathrm{C}$ following preparation. Additionally, ${ }^{89} \mathrm{Zr}$-DFO-nimotuzumab was found to transchelate in human plasma following incubation with over $20 \%$ of the radiolabel lost at $72 \mathrm{~h}$ post incubation. The pharmaceutical specification of the final ${ }^{89} \mathrm{Zr}-\mathrm{DFO}$-nimotuzumab formulation is summarized in Table 2.

${ }^{89} \mathrm{Zr}$-DFO-nimotuzumab had a $\mathrm{K}_{\mathrm{D}}$ of $14.05 \pm 2.56$ $\mathrm{nM}$ in EGFR-positive DLD-1 cells $(\sim 600,000$ EGFR/ cell) in a saturation binding analysis (Figure 2), which was similar to the binding constant obtained with A431 by flow cytometry. The immunoreactive fraction of ${ }^{89} \mathrm{Zr}-\mathrm{DFO}-$ nimotuzumab with DLD-1 cells was 73\% (Figure 2B, 2C).

\section{Pharmacokinetics, biodistribution and radiation dosimetry estimation}

${ }^{89} \mathrm{Zr}$-DFO-nimotuzumab exhibited a bi-phasic half-life with a fast (distribution) $\mathrm{t}_{1 / 2 \alpha}$ of $1.3 \mathrm{~h}$ and a slow clearance $t_{1 / 2 \beta}$ of $127.1 \mathrm{~h}$ (Figure 3 ). Biodistribution was measured in normal female (Supplementary Figure
3A) and male (Supplementary Figure 3B) mice. At early time points (up to $72 \mathrm{hp.i}$ ), there was significantly higher uptake of the tracer in bones and skin of female mice compared to male mice (Supplementary Figure 3A and $3 \mathrm{~B}$ ). However, uptake in both organs decreased to background levels at $168 \mathrm{hp}$.i. Other organs did not show differences between male and female mice. The liver had the longest residence time being the principal route of excretion of the tracer. Interestingly, the residence time in the bone marrow and skin were very short indicative of minimal transchelation of the radiometal and low skin binding, respectively (Supplementary Table 2). The liver received the highest dose (female $1.82 \mathrm{cGy} / \mathrm{mCi}$ vs male 2.64 cGy.mCi), followed by gall bladder wall (female $1.1 \mathrm{cGy} / \mathrm{mCi}$ vs male $1.3 \mathrm{cGy} / \mathrm{mCi}$ ) and osteogenic cells (female $0.9 \mathrm{cGy} / \mathrm{mCi}$ vs male $0.92 \mathrm{cGy} / \mathrm{mCi}$ ) (Table 3 ). The projected human effective dose was $0.679 \mathrm{rem} / \mathrm{mCi}$ (0.184 $\mathrm{mSv} / \mathrm{MBq})$ and $0.757 \mathrm{rem} / \mathrm{mCi}(0.205 \mathrm{mSv} / \mathrm{MBq})$ in females and males, respectively.

\section{Toxicity of ${ }^{89} \mathrm{Zr}$-DFO-nimotuzumab in mice}

Single dose acute (Day-2) and delayed (Day$14)$ toxicity was determined in normal Balb-C mice. At Day-2 p.i., there was no significant difference $(p>0.05)$ in any of the $\mathrm{CBC}$ or blood chemistry parameters in females and males compared to control (saline injected) group (Table 4A). Similarly, there was no significant difference $(p>0.05)$ in the treated compared to control group at Day-14 p.i. (Table 4B). Histopathological examination of necropsy stained slices showed no damage to the organs following administration of 168fold (radioactivity) and 25-fold (mass dose) excess of the projected human dose (data not shown).

\section{Biodistribution and microPET in tumor bearing mice}

${ }^{89} \mathrm{Zr}-\mathrm{DFO}$-nimotuzumab biodistribution was analyzed at 4, 24, 72 and 168 hp.i (Figure 4A-4D) in mice bearing xenografts with medium EGFR expression (DLD-1, MDA-MB-468) and a control negative for EGFR expression (MDA-MB-435). Tumor uptake (expressed as $\%$ injected activity per gram (\% IA/g) or $\%$ IA per volume (\% IA/cc)) in DLD-1 was slightly higher than for MDAMB-468 at $24 \mathrm{~h}(9.85 \pm 0.57$ vs $7.30 \pm 1.29 \% \mathrm{IA} / \mathrm{g})$ and 72 hp.i $(11.04 \pm 4.04$ vs $8.24 \pm 2.49 \% \mathrm{IA} / \mathrm{g})$ even though this was not significant. Control xenograft had significantly lower tumor uptake $(2.56 \pm 0.26 \% \mathrm{IA} / \mathrm{g})$ at $24 \mathrm{hp} . \mathrm{i}$. There was a high lung uptake (DLD-1: $13.82 \pm 0.57 \% \mathrm{IA} / \mathrm{g}$; MBDA-MB-468: $12.80 \pm 2.86 \% \mathrm{IA} / \mathrm{g})$ of the tracer at early time point (4 hp.i). However, this decreased to 4.79 $\pm 2.1 \% \mathrm{IA} / \mathrm{g}$ (DLD-1) and $5.61 \pm 0.49 \% \mathrm{IA} / \mathrm{g}$ (MDAMB-468) at 72 hp.i. There was also a slight increase in bone uptake of the tracer over time which is indicative of some transchelation and uptake of bone seeking free 
Table 1: Labeling efficiency and specific activity of DFO-nimotuzumab kit formulation

\begin{tabular}{lcc}
\hline Amount of antibody $(\boldsymbol{\mu g})$ per $\mathbf{1 0} \mathbf{~ M B q}$ & Labeling efficiency $\mathbf{\%} \pm \mathbf{S D}$ & Specific activity $(\mathbf{M B q} / \boldsymbol{\mu g})$ \\
\hline 5 & $21 \pm 1.1$ & 2.0 \\
10 & $86 \pm 1.0$ & 1.0 \\
20 & $91 \pm 1.2$ & 0.5 \\
50 & $92 \pm 0.4$ & 0.2 \\
100 & $94 \pm 0.9$ & 0.1 \\
\hline
\end{tabular}

Table 2: Radiopharmaceutical analyses shows the formulation meets USP specifications

\begin{tabular}{ll}
\hline Analysis & Specification \\
\hline Bubble point filter membrane integrity & $>=50 \mathrm{psi}$ \\
Visual appearance & Clear colorless solution, no visible particulate matter. \\
$\mathrm{pH}$ testing & $6.8-7.4$ \\
Strength $(\mathrm{mCi} / \mathrm{mL})$ & $1-5 \mathrm{mCi}$ in $10 \mathrm{~mL}$ \\
Radiochemical purity & iTLC $>=95 \%$ \\
Bacterial endotoxin & $<=175 \mathrm{EU} / \mathrm{V}$ \\
Specific activity & $>=0.2 \mathrm{mCi} / \mathrm{mg}$ \\
Sterility & Sterile (no visible growth) \\
& $<=1 \mathrm{cfu} / \mathrm{plate}$ (contact) \\
Environmental profile & $<=3 \mathrm{cfu} / \mathrm{plate}$ (settling) \\
Immunoreactivity & $<=3 \mathrm{cfu} /$ plate (finger) \\
\hline
\end{tabular}

${ }^{89} \mathrm{Zr}^{4+}$. The highest bone uptake observed at $72 \mathrm{~h}$ was $3.64 \pm 1.28 \% \mathrm{IA} / \mathrm{g}$. Tumor to blood ratio for DLD- 1 and MDA-MB-468 was 0.15 and 0.18 , respectively at $4 \mathrm{hp} . \mathrm{i}$ but this increased to 1.0 and 1.79 respectively at $72 \mathrm{hp} . \mathrm{i}$. Highest tumor-to-blood ratio was 2.54 at $168 \mathrm{hp.i}$ for DLD-1 tumor.

PET imaging showed persistently high tumor uptake in EGFR-positive xenografts as seen in the maximum intensity projection images of these mice at $24-168$ hp.i (Figure 5A). ${ }^{89} \mathrm{Zr}$-DFO-nimotuzumab uptake on PET was expressed as \% IA/cc (Figure 5B). There was no significant difference $(p>0.05)$ in uptake between the three EGFR-positive xenografts at 24 hp.i. At 24 hp.i uptake in DLD-1 (10.7 $\pm 0.4 \%$ IA/cc) and MDA-MB-468 $(10.9 \pm 1.3 \% \mathrm{IA} / \mathrm{cc})$ were significantly higher $(\mathrm{p}=$ $0.001)$ than in control MDA-MB-435 (7.07 $\pm 0.9 \%$ IA/ cc). At 96 and 120 hp.i, DLD-1 had the highest uptake $(\mathrm{p}<0.0001)$ compared with MDA-MB-468 xenografts. There was increasing uptake in DLD-1 and MDA-MB-468 xenografts for up to $168 \mathrm{hp} . \mathrm{i}$. The highest tumor-tobackground (muscle) ratio was observed at $168 \mathrm{hp}$.i for DLD-1 (28.4 \pm 1.1$)$ and this was significantly higher ( $\mathrm{p}$ $<0.01)$ than for MDA-MB-468 (13.8 \pm 4.2$)$ (Figure 5C). There were significant differences in tumor/muscle ratios between DLD-1 and control MDA-MB-435 at other time points. Tumor/muscle ratios were higher for MDAMB-468 compared with control MDA-MB-435 although these differences were not significant. Tumor/liver ratios were significantly higher in DLD-1 and MDA-MB-468 compared with control MDA-MB435 at all time points (Figure 5D).

\section{DISCUSSION}

EGFR is frequently overexpressed in most cancers of epithelial origin where it promotes enhanced proliferation and confers resistance to targeted therapies. Existing methods to monitor EGFR expression have yielded sub-optimal results. In addition, the expression of EGFR changes over time. It is important to develop a non-invasive method for EGFR quantitation in vivo. Nimotuzumab is an affinityoptimized antibody that is approved for squamous cell carcinoma of head \& neck, glioma, pancreatic and nasopharyngeal cancers in many jurisdictions. Other radiolabeled nimotuzumab radioimmunoconjugates including ${ }^{111}$ In-nimotuzumab and ${ }^{99 m}$ Tc-nimotuzumab have been evaluated previously in preclinical and 
A
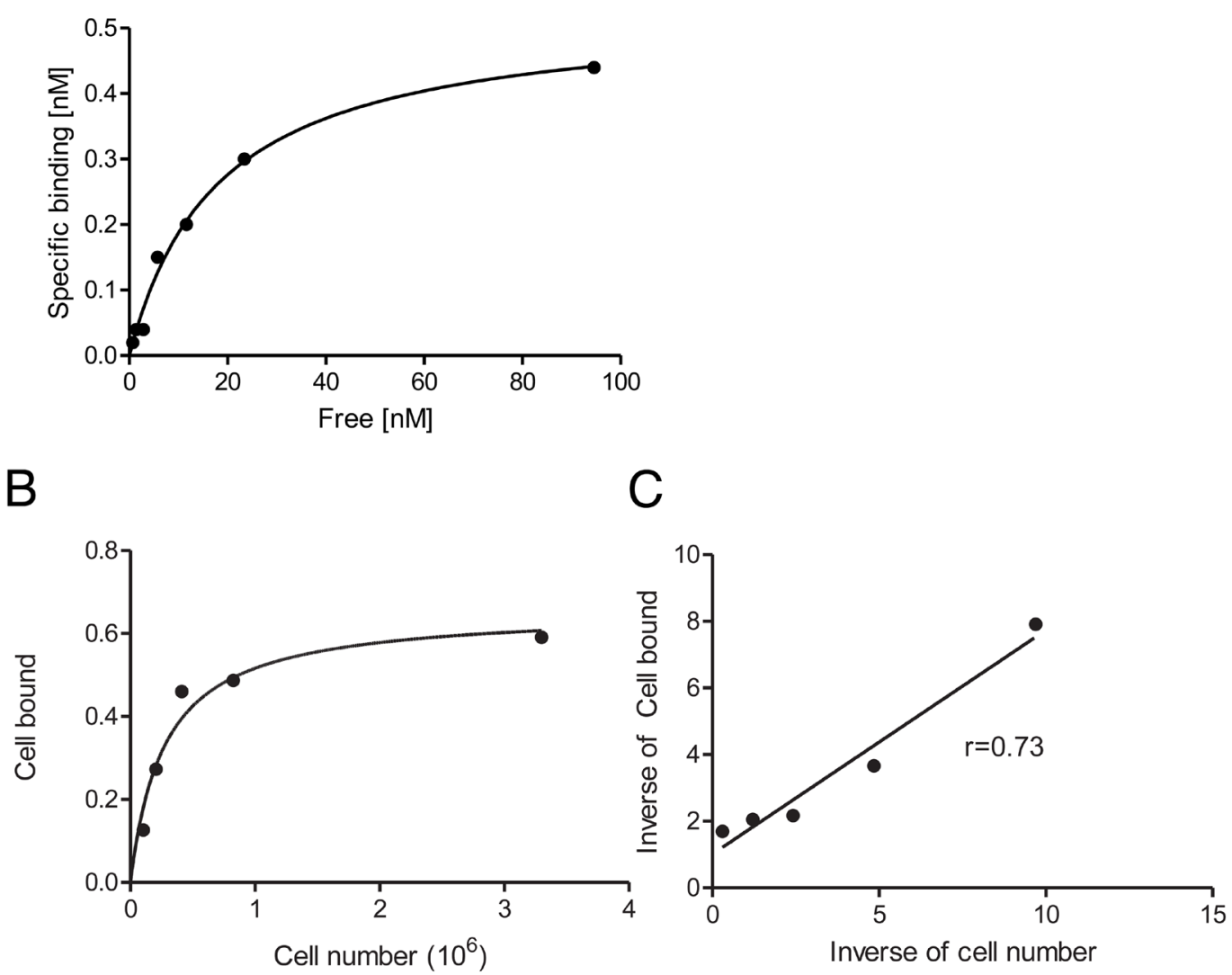

Figure 2: (A-C) Radioligand binding assay and immunoreactive fraction determination of ${ }^{89} \mathrm{Zr}$-DFO-nimotuzumab in EGFR-positive DLD-1 cells. (A) Radioligand binding assay ${ }^{89} \mathrm{Zr}$-DFO-nimotuzumab showed a $\mathrm{K}_{\mathrm{D}}$ of $14 \pm 2.4 \mathrm{nM}$ with a 577,000 EGFR/cell. (B \& C) The immunoreactive fraction of ${ }^{89} \mathrm{Zr}$-DFO-nimotuzumab was 0.73 .

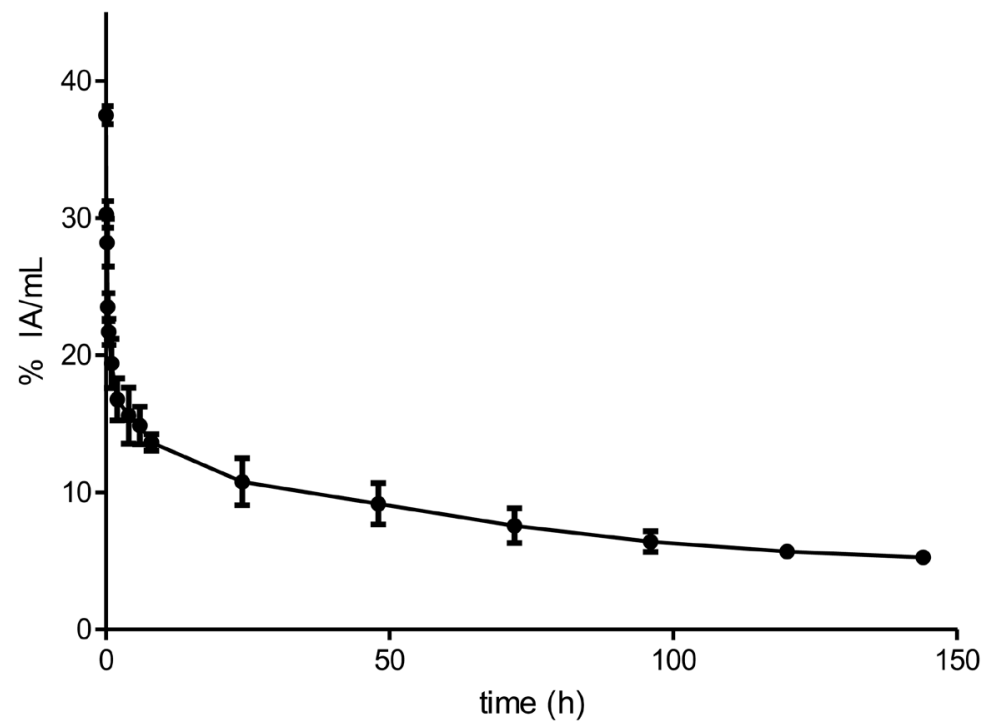

Figure 3: Pharmacokinetics of 89Zr-DFO-nimotuzumab in normal balb-C mice. ${ }^{89} \mathrm{Zr}$-DFO-nimotuzumab exhibits a biphasic kinetics with distribution and elimination half-lives of $1.3\left(\mathrm{t}_{1 / 2 \alpha}\right)$ and $127.1\left(\mathrm{t}_{1 / 2 \beta}\right)$, respectively. The volume of distribution of the central compartment (V1) was $3.2 \pm 0.3 \mathrm{~mL} \mathrm{~mL}(107 \mathrm{~mL} / \mathrm{kg})$ and the volume of distribution at steady state (Vss) was $8.6 \pm 3.4 \mathrm{~mL}(287 \mathrm{~mL} / \mathrm{kg})$. The V1 and Vss volumes correspond to $7.5 \mathrm{~L}$ and $20.1 \mathrm{~L}$ respectively, in a $70 \mathrm{~kg}$ standard adult female. The systemic clearance (CLs) was $0.045 \pm 0.0020 \mathrm{~mL} / \mathrm{h}(1.5 \mathrm{~mL} / \mathrm{h} / \mathrm{kg})$. The CLs values in mice would correspond to $105 \mathrm{~mL} / \mathrm{h}$ in a $70 \mathrm{~kg}$ adult female. 
Table 3: Projected absorbed organ doses of ${ }^{89} \mathrm{Zr}$-DFO-nimotuzumab

\begin{tabular}{|c|c|c|}
\hline Organ & Female $-{ }^{89} \mathrm{Zr}$ absorbed dose (cGy/mCi) & Male ${ }^{89} \mathrm{Zr}$ absorbed dose (cGy/mCi) \\
\hline Adrenals & 0.672 & 0.821 \\
\hline Brain & 0.205 & 0.234 \\
\hline Breasts & 0.573 & 0.601 \\
\hline Gallbladder Wall & 1.07 & 1.30 \\
\hline Lower Large Intestine & 0.757 & 0.779 \\
\hline Small Intestine & 0.929 & 0.954 \\
\hline Stomach & 0.693 & 0.783 \\
\hline Upper Large Intestine & 0.882 & 0.936 \\
\hline Heart Wall & 0.601 & 0.683 \\
\hline Kidneys & 0.603 & 0.775 \\
\hline Liver & 1.82 & 2.64 \\
\hline Lungs & 0.481 & 0.557 \\
\hline Muscle & 0.485 & 0.513 \\
\hline Ovaries & 0.656 & \\
\hline Pancreas & 0.636 & 0.819 \\
\hline Red Marrow & 0.59 & 0.624 \\
\hline Osteogenic Cells & 0.895 & 0.918 \\
\hline Skin & 0.445 & 0.462 \\
\hline Spleen & 0.431 & 1.93 \\
\hline Testes & & 0.402 \\
\hline Thymus & 0.452 & 0.473 \\
\hline Thyroid & 0.618 & 0.623 \\
\hline Urinary Bladder & 0.639 & 0.643 \\
\hline Uterus & 0.564 & \\
\hline Total Body & 0.624 & 0.677 \\
\hline Effective dose (rem/mCi) & 0.679 & 0.757 \\
\hline
\end{tabular}

early-phase clinical trials $[10,15]$. While SPECT agents labeled with ${ }^{111} \mathrm{In}$ and ${ }^{99 \mathrm{~m}} \mathrm{Tc}$ may offer some advantages over other PET isotopes such as lower radiation dose exposure, the inherent image quality and quantitative capabilities of PET is superior to SPECT.

Here, we evaluated the PET imaging properties of ${ }^{89} \mathrm{Zr}$-DFO-nimotuzumab. First, we developed a kit preparation of DFO-nimotuzumab in accordance with USP standards. Several batches of DFO-conjugated nimotuzumab have been produced to date and all have met and exceeded USP specifications (Table 2). Importantly, the conjugation of DFO did not significantly alter the binding of nimotuzumab to EGFR-positive cells and the shelf-life of DFO-nimotuzumab was 6 months at $4^{\circ} \mathrm{C}$ and even longer at $-80^{\circ} \mathrm{C}$.
${ }^{89} \mathrm{Zr}$-DFO-nimotuzumab did not cause any changes in $\mathrm{CBC}$ and blood chemistry following injection of excess mass and radioactivity dose of the projected human dose of the tracer $-74 \mathrm{MBq} 10 \mathrm{mg}$. The projected effective dose in humans was $0.679 \mathrm{rem} / \mathrm{mCi}(0.184 \mathrm{mSv} / \mathrm{MBq}$ in female and $0.757 \mathrm{rem} / \mathrm{mCi}(0.205 \mathrm{mSv} / \mathrm{MBq})$ in males. There are several immunoPET imaging agents in clinical trial, notably, anti-Her2 ${ }^{89} \mathrm{Zr}$-DFO-trastuzumab and anti-EGFR ${ }^{89} \mathrm{Zr}$-DFO-cetuximab are in advanced development. The effective dose of ${ }^{89} \mathrm{Zr}$-DFO-trastuzumab as determined by Laforest et al. was $0.61 \mathrm{mSv} / \mathrm{MBq}$. Similarly, Bhattacharyya et al. determined the effective dose of ${ }^{89} \mathrm{Zr}$-DFO-panitumumab was $0.57 \mathrm{mSv} / \mathrm{mCi}$. The effective dose ${ }^{89} \mathrm{Zr}$-DFO-nimotuzumab was 3.3fold and 3.1-fold lower than for ${ }^{89} \mathrm{Zr}$-DFO-trastuzumab 
Table 4A: Acute (2-day) toxicity of ${ }^{89} \mathrm{Zr}$-DFO-nimotuzumab

\begin{tabular}{|c|c|c|c|c|}
\hline \multirow{2}{*}{$\begin{array}{l}\text { Parameters } \\
\text { Clinical chemistry }\end{array}$} & \multicolumn{2}{|c|}{ Male \pm Std Dev. ${ }^{*}$} & \multicolumn{2}{|c|}{ Female \pm Std Dev. } \\
\hline & Control treated & $\begin{array}{c}{ }^{89} \mathrm{Zr} \text {-DFO- } \\
\text { nimotuzumab }\end{array}$ & Control treated & $\begin{array}{c}{ }^{89} \mathrm{Zr} \text {-DFO- } \\
\text { nimotuzumab }\end{array}$ \\
\hline $\begin{array}{l}\text { Albumin:Globulin } \\
(\mathrm{g} / \mathrm{L})\end{array}$ & $1.61 \pm 0.13$ & $1.51 \pm 0.05$ & $2.08 \pm 0.13$ & $2.01 \pm 0.22$ \\
\hline Albumin $(\mathrm{g} / \mathrm{L})$ & $29.21 \pm 1.09$ & $29.0 \pm 0.70$ & $32.8 \pm 1.48$ & $32.0 \pm 1.41$ \\
\hline Globulin $(\mathrm{g} / \mathrm{L})$ & $18.2 \pm 0.84$ & $19.2 \pm 0.44$ & $15.8 \pm 1.09$ & $16.0 \pm 1.22$ \\
\hline $\begin{array}{l}\text { Alkaline Phosphatase } \\
\text { (U/L) }\end{array}$ & $146.41 \pm 11.39$ & $139.60 \pm 9.91$ & $147.20 \pm 6.38$ & $140.80 \pm 2.59$ \\
\hline $\operatorname{ALT}(\mathrm{U} / \mathrm{L})$ & $24.21 \pm 2.58$ & $25.81 \pm 1.3$ & $25.80 \pm 5.76$ & $26.60 \pm 4.51$ \\
\hline AST (U/L) & $69.81 \pm 9.85$ & $83.21 \pm 17.98$ & $116.4 \pm 82.50$ & $73.0 \pm 15.02$ \\
\hline Bicarbonate (mmol/L) & $17.01 \pm 0.7$ & $17.41 \pm 1.14$ & $15.4 \pm 2.97$ & $18.0 \pm 0.70$ \\
\hline $\mathrm{Ca}^{2+}(\mathrm{mmol} / \mathrm{L})$ & $2.48 \pm 0.07$ & $2.39 \pm 0.14$ & $2.28 \pm 0.04$ & $2.37 \pm 0.13$ \\
\hline $\mathrm{Cl}^{-1}(\mathrm{mmol} / \mathrm{L})$ & $109.20 \pm 1.483$ & $108.2 \pm 0.83$ & $106.0 \pm 4.12$ & $108.0 \pm 2.12$ \\
\hline Creatinine & $14.6 \pm 1.67$ & $12.8 \pm 2.59$ & $18.0 \pm 5.43$ & $14.41 \pm 3.05$ \\
\hline Glucose (mmol/L) & $13.68 \pm 1.70$ & $12.68 \pm 1.29$ & $14.02 \pm 1.72$ & $12.50 \pm 1.11$ \\
\hline $\mathrm{K}+(\mathrm{mmol} / \mathrm{L})$ & $5.12 \pm 0.55$ & $6.48 \pm 0.69$ & $5.12 \pm 0.85$ & $5.1 \pm 0.73$ \\
\hline $\mathrm{Na}+(\mathrm{mmol} / \mathrm{L})$ & $147.8 \pm 1.79$ & $145.0 \pm 1.58$ & $142.20 \pm 4.44$ & $144.2 \pm 1.92$ \\
\hline $\mathrm{Na}+/ \mathrm{Ka}+$ & $29.41 \pm 2.9$ & $22.4 \pm 2.4$ & $28.40 \pm 4.80$ & $28.8 \pm 4.87$ \\
\hline $\begin{array}{l}\text { Total bilirubin } \\
(\mu \mathrm{mol} / \mathrm{L})\end{array}$ & $1.28 \pm 0.39$ & $1.42 \pm 0.39$ & $0.36 \pm 0.33$ & $0.96 \pm 0.15$ \\
\hline Total protein $(\mathrm{g} / \mathrm{L})$ & $47.4 \pm 0.54$ & $48.2 \pm 0.83$ & $48.6 \pm 2.19$ & $48.0 \pm 1.4$ \\
\hline Urea $(\mathrm{mmol} / \mathrm{L})$ & $6.86 \pm 0.6$ & $7.30 \pm 0.89$ & $6.86 \pm 0.6$ & $7.30 \pm 0.88$ \\
\hline \multicolumn{5}{|l|}{ Cell Blood Counts } \\
\hline $\mathrm{HCT}(\mathrm{L} / \mathrm{L})$ & $0.47 \pm 0.05$ & $0.47 \pm 0.02$ & $0.48 \pm 0.02$ & $0.48 \pm 0.01$ \\
\hline $\operatorname{HGB}(\mathrm{g} / \mathrm{L})$ & $144.0 \pm 12.73$ & $144.2 \pm 5.77$ & $148.20 \pm 3.96$ & $150.40 \pm 3.05$ \\
\hline $\mathrm{MCH}(\mathrm{pg})$ & $15.05 \pm 0.19$ & $14.9 \pm 0.22$ & $15.21 \pm 0.10$ & $15.18 \pm 0.16$ \\
\hline $\mathrm{MCHC}(\mathrm{g} / \mathrm{L})$ & $307.3 \pm 4.99$ & $307.6 \pm 3.8$ & $309.80 \pm 3.63$ & $315.0 \pm 5.29$ \\
\hline $\mathrm{MCV}(\mathrm{fL})$ & $48.93 \pm 0.3$ & $48.42 \pm 0.44$ & $48.96 \pm 0.48$ & $48.14 \pm 0.42$ \\
\hline $\mathrm{RBC}\left(\mathrm{x} 10^{12} / \mathrm{L}\right)$ & $9.55 \pm 0.92$ & $9.68 \pm 0.37$ & $9.76 \pm 0.3$ & $9.92 \pm 0.23$ \\
\hline RDW (\%) & $14.35 \pm 0.47$ & $14.26 \pm 0.47$ & $14.98 \pm 0.41$ & $14.90 \pm 0.32$ \\
\hline WBC (x109/L) & $2.45 \pm 1.59$ & $2.9 \pm 1.50$ & $3.06 \pm 0.90$ & $2.08 \pm 0.64$ \\
\hline
\end{tabular}

${ }^{*}$ (male) and ${ }^{* *}$ (female): There were no significant differences in all parameters between control treated and ${ }^{89} \mathrm{Zr}$-DFOnimotuzumab treated mice following injection of excess of ${ }^{89} \mathrm{Zr}$-DFO-nimotuzumab.

and ${ }^{89} \mathrm{Zr}$-DFO-panitumumab, respectively $[16,17]$. The lower absorbed dose of ${ }^{89} \mathrm{Zr}$-DFO-nimotuzumab to organs offers the possibility of administering multiple doses and remain within the lower limit of organ dose set by the FDA. Radioactive Drug Research Committee (FDA RDRC) guidelines, which stipulates that for whole body, 
Table 4B: Acute (14-day) toxicity of ${ }^{89} \mathrm{Zr}$-DFO-nimotuzumab

\begin{tabular}{|c|c|c|c|c|}
\hline \multirow{2}{*}{$\frac{\text { Parameters }}{\text { Clinical chemistry }}$} & \multicolumn{2}{|c|}{ Male \pm Std Dev. } & \multicolumn{2}{|c|}{ Female \pm Std Dev } \\
\hline & Control treated & $\begin{array}{c}{ }^{89} \mathrm{Zr}-\mathrm{DFO}- \\
\text { nimotuzumab }\end{array}$ & Control treated & $\begin{array}{c}{ }^{89} \mathrm{Zr}-\mathrm{DFO}- \\
\text { nimotuzumab }\end{array}$ \\
\hline $\begin{array}{l}\text { Albumin:Globulin } \\
(\mathrm{g} / \mathrm{L})\end{array}$ & $1.37 \pm 0.23$ & $1.49 \pm 0.07$ & $2.19 \pm 0.21$ & $1.94 \pm 0.15$ \\
\hline Albumin $(\mathrm{g} / \mathrm{L})$ & $27.40 \pm 3.36$ & $29.0 \pm 1.41$ & $31.80 \pm 0.84$ & $31.40 \pm 28.43$ \\
\hline Globulin $(\mathrm{g} / \mathrm{L})$ & $20.21 \pm 1.64$ & $19.4 \pm 0.89$ & $14.60 \pm 1.14$ & $16.2 \pm 0.83$ \\
\hline $\begin{array}{l}\text { Alkaline Phosphatase } \\
(\mathrm{U} / \mathrm{L})\end{array}$ & $106.60 \pm 11.72$ & $108.2 \pm 4.42$ & $127.60 \pm 2.30$ & $115.6 \pm 4.61$ \\
\hline $\operatorname{ALT}(\mathrm{U} / \mathrm{L})$ & $35.4 \pm 8.79$ & $27.40 \pm 1.14$ & $26.20 \pm 3.56$ & $23.60 \pm 2.70$ \\
\hline AST (U/L) & $233.4 \pm 143.3$ & $71.0 \pm 11.29$ & $78.80 \pm 12.48$ & $103.80 \pm 28.43$ \\
\hline Bicarbonate (mmol/L) & $15.81 \pm 2.49$ & $18.4 \pm 1.51$ & $15.21 \pm 1.48$ & $16.01 \pm 1.58$ \\
\hline $\mathrm{Ca}^{2+}(\mathrm{mmol} / \mathrm{L})$ & $2.35 \pm 0.13$ & $2.36 \pm 0.10$ & $2.27 \pm 0.09$ & $2.27 \pm 0.03$ \\
\hline $\mathrm{Cl}^{-1}(\mathrm{mmol} / \mathrm{L})$ & $104.6 \pm 3.36$ & $106.6 \pm 1.52$ & $107.2 \pm 2.58$ & $107.0 \pm 1.58$ \\
\hline Creatinine & $13.4 \pm 1.82$ & $14.60 \pm 2.70$ & $12.8 \pm 0.84$ & $13.8 \pm 2.0$ \\
\hline Glucose (mmol/L) & $12.22 \pm 1.37$ & $11.89 \pm 0.61$ & $11.42 \pm 0.85$ & $11.62 \pm 2.27$ \\
\hline $\mathrm{K}+(\mathrm{mmol} / \mathrm{L})$ & $6.20 \pm 1.06$ & $5.18 \pm 0.33$ & $4.52 \pm 0.47$ & $4.30 \pm 0.50$ \\
\hline $\mathrm{Na}+(\mathrm{mmol} / \mathrm{L})$ & $147.40 \pm 4.39$ & $149.6 \pm 1.52$ & $148.0 \pm 1.87$ & $147.4 \pm 0.89$ \\
\hline $\mathrm{Na}+/ \mathrm{Ka}+$ & $24.40 \pm 5.03$ & $29.0 \pm 2.0$ & $32.81 \pm 4.03$ & $34.80 \pm 3.7$ \\
\hline $\begin{array}{l}\text { Total bilirubin } \\
(\mu \mathrm{mol} / \mathrm{L})\end{array}$ & $1.32 \pm 0.33$ & $0.62 \pm 0.24$ & $0.82 \pm 0.18$ & $1.02 \pm 0.33$ \\
\hline Total protein $(\mathrm{g} / \mathrm{L})$ & $47.6 \pm 2.4$ & $48.4 \pm 1.95$ & $46.41 \pm 1.14$ & $47.60 \pm 0.54$ \\
\hline Urea (mmol/L) & $7.48 \pm 0.39$ & $9.52 \pm 0.93$ & $7.48 \pm 0.39$ & $9.52 \pm 0.93$ \\
\hline \multicolumn{5}{|l|}{ Cell Blood Counts } \\
\hline $\mathrm{HCT}(\mathrm{L} / \mathrm{L})$ & $0.46 \pm 0.02$ & $0.47 \pm 0.03$ & $0.47 \pm 0.01$ & $0.47 \pm 0.01$ \\
\hline $\operatorname{HGB}(\mathrm{g} / \mathrm{L})$ & $147.40 \pm 5.22$ & $148.3 \pm 6.75$ & $148.60 \pm 4.04$ & $150.60 \pm 4.34$ \\
\hline $\mathrm{MCH}(\mathrm{pg})$ & $15.04 \pm 0.20$ & $15.35 \pm 0.13$ & $15.30 \pm 0.14$ & $15.66 \pm 0.27$ \\
\hline $\mathrm{MCHC}(\mathrm{g} / \mathrm{L})$ & $317.40 \pm 5.68$ & $311.30 \pm 7.6$ & $313.80 \pm 5.93$ & $318.4 \pm 6.22$ \\
\hline MCV (fL) & $47.46 \pm 0.41$ & $49.33 \pm 1.68$ & $48.76 \pm 0.65$ & $49.18 \pm 0.59$ \\
\hline $\mathrm{RBC}\left(\mathrm{x} 10^{12} / \mathrm{L}\right)$ & $9.8 \pm 0.41$ & $9.66 \pm 0.49$ & $9.71 \pm 0.25$ & $9.61 \pm 0.33$ \\
\hline RDW (\%) & $13.66 \pm 0.11$ & $14.73 \pm 0.29$ & $14.24 \pm 0.25$ & $14.64 \pm 0.25$ \\
\hline WBC (x109/L) & $1.92 \pm 1.22$ & $2.18 \pm 0.71$ & $2.14 \pm 1.12$ & $1.25 \pm 0.06$ \\
\hline
\end{tabular}

${ }^{*}$ (male) and ${ }^{* *}$ (female): There were no significant differences in all parameters between control treated and ${ }^{89} \mathrm{Z}-\mathrm{DFO}-$ nimotuzumab treated mice following injection of excess of ${ }^{89} \mathrm{Zr}$-DFO-nimotuzumab.

active blood-forming organs, lens of eye, and gonads, the single dose must not exceed $30 \mathrm{mCv}$ (3 rems) or annual dose of $50 \mathrm{mSv}$ ( $5 \mathrm{rems}$ ). In line with the FDA RDRC guidelines, patients on ${ }^{89} \mathrm{Zr}$-DFO-trastuzumab and ${ }^{89} \mathrm{Zr}$ DFO-cetuximab clinical trials were infused an average dose of $37 \mathrm{MBq}$. A $37 \mathrm{MBq}$ dose corresponds to a 22.57 $\mathrm{mSv}$ and $21.1 \mathrm{mSv}$ effective dose for ${ }^{89} \mathrm{Zr}$-trastuzumab and ${ }^{89} \mathrm{Zr}$-cetuximab, respectively. However, administration of
$37 \mathrm{MBq}$ of ${ }^{89} \mathrm{Zr}$-nimotuzumab will result in an estimated dose of only $6.81 \mathrm{mSv}$. By comparison, the estimated dose of ${ }^{18} \mathrm{~F}-\mathrm{FDG}$, the most widely used PET imaging agent is $7.4 \mathrm{mSv}$ for an adult patient receiving $370-$ $540 \mathrm{MBq}$ [18]. Assuming the same (37 MBq) patient dose as was infused for the other immunoPET agents the administration of ${ }^{89} \mathrm{Zr}$-DFO-nimotuzumab would result in scans of diagnostic quality that offers a similar 
or lower dose than FDG. Similar to FDG, the low body dose to patients of ${ }^{89} \mathrm{Zr}$-DFO-nimotuzumab could allow for repeated dosing or follow up scans that cannot be done with other immunoPET agents such as ${ }^{89} \mathrm{Zr}$-DFOcetuximab. This low observed toxicity was confirmed by $\mathrm{CBC}$ and blood biochemistry studies which showed no significant difference in all blood cells and chemistry parameters investigated.

${ }^{89} \mathrm{Zr}$-DFO-nimotuzumab showed early high uptake in tumor xenografts. Tumor uptake was up to $6.2 \%$ IA/cc at $4 \mathrm{hp} . \mathrm{i}$ and this uptake increased and remain persistently high at the end of imaging $168 \mathrm{hp} . \mathrm{i}$ where the highest uptake was observed in DLD-1 xenograft (18.3\%IA/cc). Tumors were clearly delineated at 24 hp.i with the highest tumor to muscle ratios observed at 168 hp.i. Early delineation of tumor may be possible in patients with ${ }^{89} \mathrm{Zr}$-DFO-nimotuzumab given the early high tumor uptake and the rapid off-target clearance of the tracer (as evidence from the lower organ doses). IgGs have not been the molecule of choice for ImmunoPET because of the slow accumulation, slow blood clearance, and high background. As a result, images of diagnostic quality have mostly been obtained at $4-7$ days p.i.. In the Even et al. study, the recommended imaging time using ${ }^{89} \mathrm{Zr}$-DFO-cetuximab for patients with colorectal cancer was $6-7$ day p.i. [19]. Similarly, in the study by Dijkers et al. the best time for assessment of ${ }^{89} \mathrm{Zr}$-trastuzumab uptake by tumors was 4-5 days [20].

We developed a clinical-grade ${ }^{89} \mathrm{Zr}-\mathrm{DFO}-$ nimotuzumab as a potential immunoPET agent for imaging of patients with EGFR-positive cancers. The kit formulation DFO-nimotuzumab and imaging agent ${ }^{89} \mathrm{Zr}$ DFO-nimotuzumab met the USP and Health Canada QC requirements for an investigational agent. The projected human effective dose is more than three-fold lower than for similar immunoPET imaging agents in clinical trials. At a human dose of $37 \mathrm{MBq}(10 \mathrm{mg})$ the effective dose for ${ }^{89} \mathrm{Zr}$-DFO-nimotuzumab would be comparable to that of ${ }^{18} \mathrm{~F}$-FDG. Our results showed that ${ }^{89} \mathrm{Zr}$-DFO-nimotuzumab
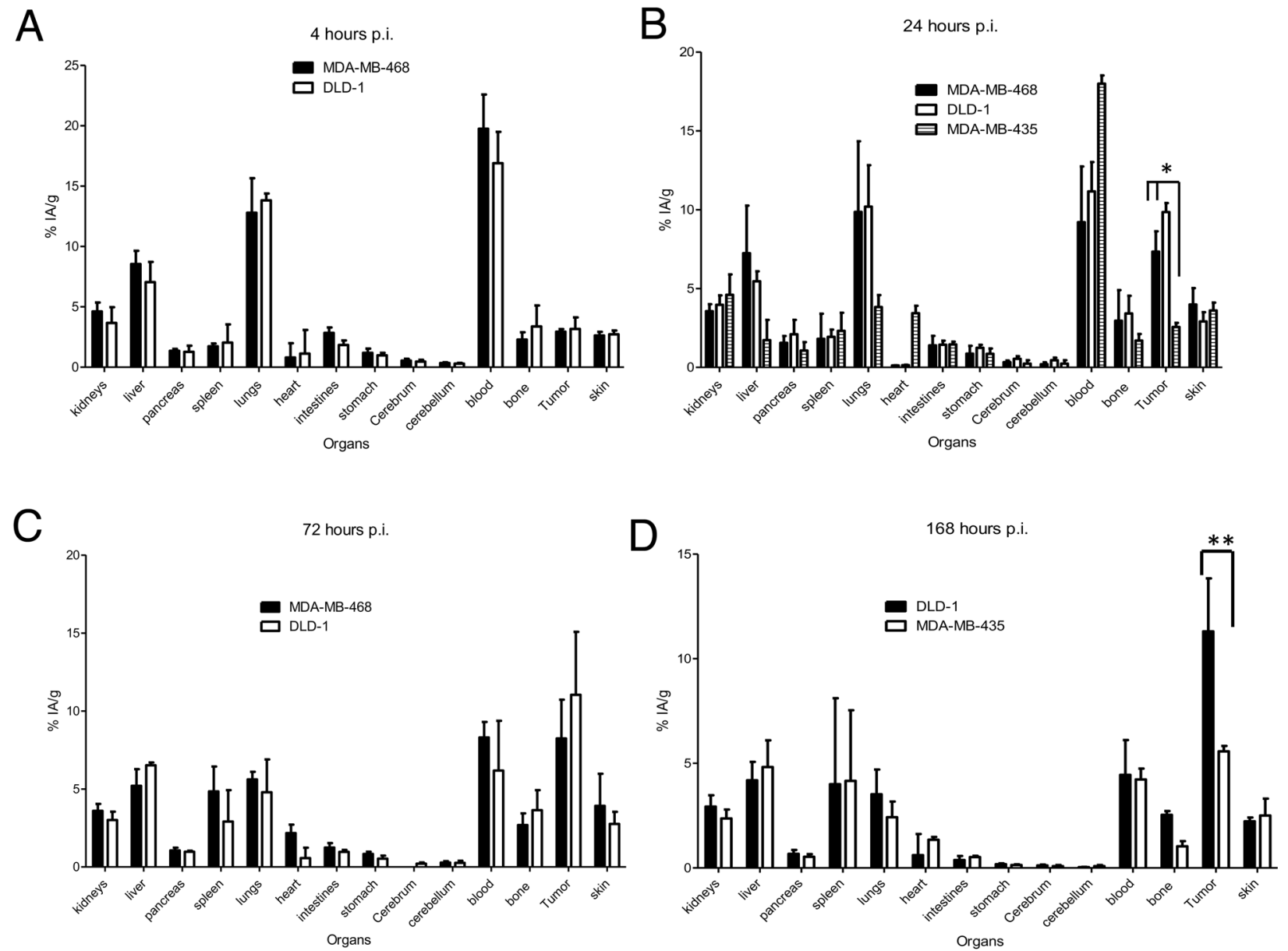

Figure 4: (A-D) Biodistribution of ${ }^{89} \mathrm{Zr}$-DFO-nimotuzumab in EGFR-positive MDA-MB-468, DLD-1, and control (very low EGFR expressing) MDA-MB-435 xenografts at 4, 24, 72 and $168 \mathrm{~h}$ post injection. Athymic CD-1 nude mice xenografts were injected via a tail vein with $10 \mathrm{MBq} 10 \mu \mathrm{g}$ of ${ }^{89} \mathrm{Zr}$-DFO-nimotuzumab followed by biodistribution studies. Uptake in MDA-MB-468 and DLD-1 tumors was significantly higher than in control MDA-MB-435 at $24\left({ }^{*} p<0.05\right)$ and $168 \mathrm{~h}\left({ }^{* *} p<0.05\right)$ post injection. 
A

DLD-1

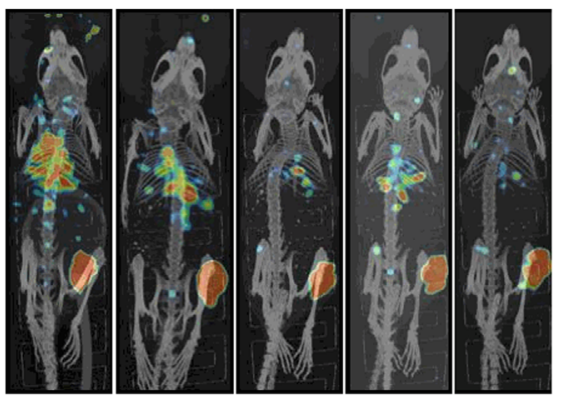

$24 \mathrm{~h}$

$72 \mathrm{~h}$

$120 \mathrm{~h} \quad 144 \mathrm{~h} \quad 168 \mathrm{~h}$

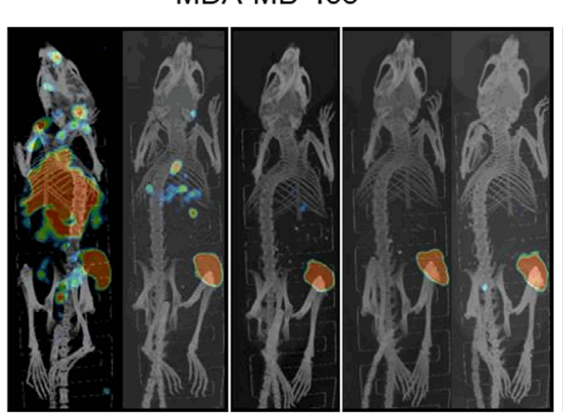

$24 \mathrm{~h} \quad 72 \mathrm{~h} \quad 120 \mathrm{~h} \quad 144 \mathrm{~h} 168 \mathrm{~h}$

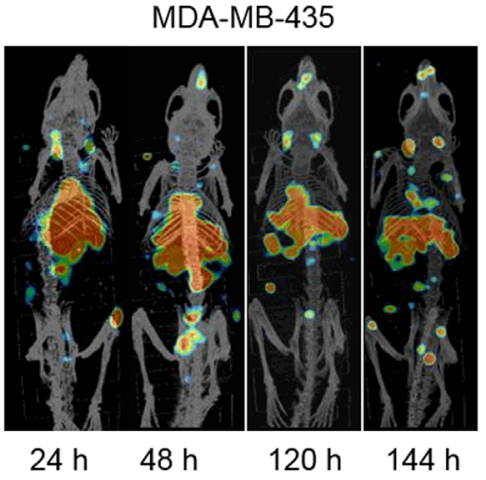

$\mathrm{B}$
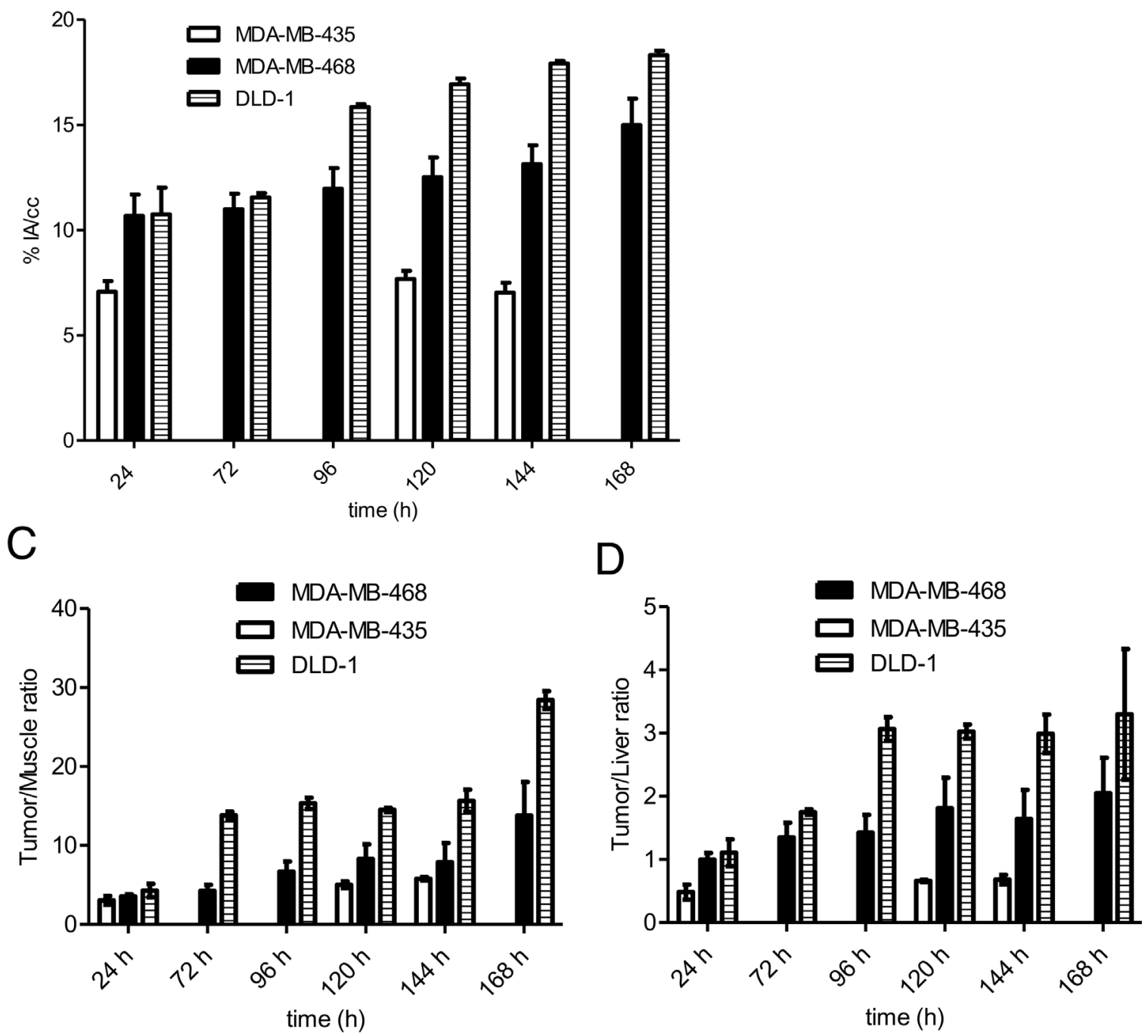

Figure 5: (A-D) PET/CT imaging and image analyses in mice xenografts. (A) Maximum intensity projection (MIP) PET/CT images of a representative mouse bearing EGFR-positive DLD-1 and MDA-MB-468, and control MDA-MB-435 xenografts at different time points post ${ }^{89} \mathrm{Zr}$-DFO-nimotuzumab injection. Xenografts are located in right thigh of the hind leg. (B) Except at 24 h post injection, significantly higher tumor uptake was seen in EGFR xenografts compared with control xenograft. (C) Tumor/muscle and (D) Tumor/liver ratios in same mice groups. 
could provide earlier tumor delineation and with low organ radiation dose.

\section{MATERIALS AND METHODS}

The conjugation of nimotuzumab to DFO and labeling with ${ }^{89} \mathrm{Zr}$ were performed following good manufacturing practice (GMP) conditions at the Saskatchewan Centre for Cyclotron Sciences (SCCS). Materials and solvents for the labeling procedure were sterile and endotoxin and metal free except for Amicon Ultra-4 Centrifugal Filter 30K NMCO, (EMD Millipore). Protein concentration, endotoxin content, and sterility were measured. All chemicals used for the study complied with U.S. Pharmacopoeia specifications. Clinical-grade nimotuzumab was obtained from the Center for Molecular Immunology (CIM: Havana) and was used as per terms of the research collaboration and material transfer.

\section{Cell lines and xenografts}

Human cancer cell lines MDA-MB-468, DLD-1, and A431 that express EGFR and negative control (no EGFR expression) MDA-MB-435 cell line were obtained from ATCC (Rockville, MD, USA). Cells were propagated by serial passage in MEM/EBSS medium, supplemented with $10 \%$ fetal bovine serum (Biochrom) at $37^{\circ} \mathrm{C}$ in a humidified atmosphere of $5 \% \mathrm{CO}_{2}$.

All animals used in imaging experiments were cared for and maintained under the supervision and guidelines of the University of Saskatchewan Animal Care Committee (protocol \# 20160005). Female CD-1 nude mice were obtained from Charles River Canada (St-Constant, Quebec) at 4 weeks of age and housed in a $12 \mathrm{~h}$ light, $12 \mathrm{~h}$ dark cycle in a temperature and humidity controlled vivarium. Animals had libitum access to mouse diet (Lab Diet, St. Louis, Missouri) and water. After one week of acclimatization, mice were subcutaneously injected with a suspension of 5-10 × $10^{6}$ MDA-MB-468, DLD-1, A431 or MDA-MB-435 cells in $100 \mu \mathrm{L}$ of a 1:1 mixture of serum-free MEM/EBSS medium (HyClone Laboratories, Logan, Utah) and Matrigel matrix basement membrane (Discovery Laboware, Inc. Bedford, MA) at the right? hind limb of each mouse. Tumor growth was followed with caliper measurements.

\section{Conjugation of nimotuzumab with p-SCN-Bz- desferoxamine}

Nimotuzumab (5 $\mathrm{mg} / \mathrm{mL}$ ) in PBS was buffer exchanged in $0.1 \mathrm{M} \mathrm{NaHCO}_{3}(\mathrm{pH} 9)$ using centrifugal filters (Amicon Ultra-4 Centrifugal Filter 30K NMCO, EMD Millipore) and concentrated to $10 \mathrm{mg} / \mathrm{mL}$ nimotuzumab in the bicarbonate solution [21]. A tenfold mole excess of p-SCN-Bz-Deferoxamine (DFO: Macrocyclics) in DMSO was added dropwise to the antibody solution (final volume of DMSO was kept below $5 \%$ ). The final concentration of the reaction mixture was $9.5 \mathrm{mg} / \mathrm{mL}$. The reaction mixture was incubated at $37^{\circ} \mathrm{C}$ on a shaker at 650 RPM for an hour. The reaction mixture was cooled to room temperature and the unreacted DFO was removed by two consecutive centrifugations with desalting columns $\left(\mathrm{Zeba}^{\mathrm{TM}}\right.$ Spin Desalting Columns, 7K MWCO, Thermofisher). The buffer was exchanged with PBS using centrifugation with centrifugal filters and sterilized with $0.2 \mu \mathrm{m}$ hydrophilic PTFE membrane filters (Ultrafree ${ }^{\circledR}-C L$ Centrifugal filters, Millipore). The solution of DFO-nimotuzumab was aliquoted and stored at $-80^{\circ} \mathrm{C}$ until further use.

\section{Quality control of DFO-nimotuzumab}

Nimotuzumab and DFO-nimotuzumab purity was determined using SEC-HPLC (Waters 2796 Bioseparations Module, Waters 2487 Dual $\lambda$ Absorbance Detector, XBridge ${ }^{\circledR}$ BEH 200A SEC $3.5 \mu \mathrm{m} 7.8 \times 300 \mathrm{~mm}$ column, Waters Corporation). The UV-Detector was set at 220 and $280 \mathrm{~nm}$ and the solvent system was PBS at a flow rate of $0.6 \mathrm{~mL} / \mathrm{min}$.

The analysis of MW and purity of conjugated samples were performed on an Agilent 2100 Bioanalyzer using Agilent High Sensitivity Protein 250 Kit) using manufacturer's protocol. The ladder or antibody solution in non-reducing buffer was heated to $95^{\circ} \mathrm{C}$ for 5 minutes before analysis. The size and relative peak area were calculated using Agilent 2100 Expert software. The difference in molecular weight between the nimotuzumab and DFO-nimotuzumab was used to determine the average number of chelator (DFO) on the antibody.

Binding kinetics between the antibodies and EGFR were measured using BLI with ForteBio Octet RED384 (PALL Corporation). Antibodies were immobilized on Anti-human FAB-CH1 sensors (18-5104, Forte Bio) according to manufactures instructions. The equilibrium dissociation constant $\left(\mathrm{K}_{\mathrm{D}}\right)$ was obtained using a 1 to 1 binding model with global fitting. Data analysis and curve fitting was performed using Data Analysis software 7.1.0.33 (Forte Bio).

The sterility of nimotuzumab and DFOnimotuzumab was measured according to USP 71 and bacterial endotoxin test, according to USP 85. Thioglycollate, Trypone Soy Broth, Tryptic Soy Agar (TS) media were used as culture media. For endotoxin limit test, the FDA approved Endosafe-Portable Test System (Charles River Laboratories) was used.

\section{In vitro binding assay by flow cytometery}

A431 cells were collected and washed with PBS $+2 \%$ FBS. nimotuzumab and DFO-nimotuzumab were titrated at a minimum of a 10-fold excess onto cells (twelve concentration points starting from $2 \mu \mathrm{M}$ ). Cells with 
nimotuzumab and DFO-nimotuzumab were incubated for 30 minutes at room temperature followed by 15 minutes on ice. Cells were washed and suspended in a 1:50 dilution of FITC labelled Goat F(ab')2 fragment anti-human IgG $(\mathrm{H}+\mathrm{L})$ antibody (Beckman Coulter) and incubated for 30 minutes on ice in the dark. Cells were washed and suspended in PBS + $2 \%$ FBS and analyzed using a Gallios flow cytometer (Beckman Coulter) on the FL1 channel.

\section{${ }^{89} \mathrm{Zr}$ radiolabeling of DFO-nimotuzumab}

1 M Hepes pH 7.4 (Fisher Scientific) was added to ${ }^{89} \mathrm{Zr}$ in oxalic acid (Washington University School of Medicine in St. Louise) and $2 \mathrm{M} \mathrm{NaCO}_{3}$ (pH 11) was added drop wise while measuring the $\mathrm{pH}$ with $\mathrm{pH}$ paper until the oxalic acid was neutralized $(\mathrm{pH} 7 \pm 0.2)$. DFOnimotuzumab was then added to the ${ }^{89} \mathrm{Zr}$ solution at five different specific activities $(2,1,0.5,0.2$ and $0.1 \mathrm{MBq} / \mu \mathrm{g})$. The reaction mixture was incubated at $37^{\circ} \mathrm{C}$ on a shaker at 650 RPM for two hours. The reaction mixture was cooled to room temperature and an aliquot was injected onto SECHPLC (the same HPLC and solvent system as above) for radiochemical quality control and radiolabeling efficiency determination. The eluate from the SEC-HPLC was collected to determine the recovery of radioactivity. Oxalate, hepes and other impurities (less than $30 \mathrm{kDa}$ ) were removed from crude reaction mixture by single centrifugation in spin columns (Amicon Ultra-4 Centrifugal Filter 30K NMCO, $4 \mathrm{~mL}$, EMD Millipore) and sterilized by filtration with $0.2 \mu \mathrm{m}$ hydrophilic PTFE membrane filter (Ultrafree ${ }^{\circledR}$-CL Centrifugal filters $4 \mathrm{~mL}$, Millipore). The final solution was formulated in PBS. The chemical and radiochemical purity of final solution was determined by SEC-HPLC. ${ }^{89} \mathrm{Zr}-\mathrm{DFO}-$ nimotuzmab with a radiochemical purity of more than $90 \%$ was used for in vitro and in vivo experiments.

The storage stability (shell life) of nimotuzumab and DFO-nimotuzumab at $-80^{\circ} \mathrm{C}$ and $4{ }^{\circ} \mathrm{C}$ (quadruplets for each) was determined by analyzing aliquots from each sample by SEC-HPLC. An aliquot was thawed from $-80^{\circ} \mathrm{C}$ on a monthly basis and analyzed by HPLC to determine the purities of the solution over time compared to the initial purities of the immunoconjugates. The stability of ${ }^{89} \mathrm{Zr}$ DFO-nimotuzumab was evaluated in solution $(0.9 \% \mathrm{NaCl})$ and in human plasma at $37^{\circ} \mathrm{C}$ for $7 \mathrm{~d}(\mathrm{~N}=3) .{ }^{89} \mathrm{Zr}-\mathrm{DFO}-$ nimotuzumab was added to $0.9 \% \mathrm{NaCl}$ or human plasma to make a final concentration of $18 \mathrm{MBq} / \mathrm{mL}$. An aliquot at different time points and up to 7 days were drawn and analyzed for radiochemical purity with SEC-HPLC. An aliquot of ${ }^{89} \mathrm{Zr}$-DFO-nimotuzumab (triplicates) stored at room temperature and $4^{\circ} \mathrm{C}$ was determined at $2,6,16,24$, and $48 \mathrm{~h}$ post incubation and purification by SEC-HPLC.

\section{Radioligand saturation binding assay and immunoreactive fraction determination}

The binding of ${ }^{89} \mathrm{Zr}-\mathrm{DFO}$-nimotuzumab to EGFR-positive DLD-1 cells was determined using a saturation radioligand binding assay by incubating increasing concentrations of radio-immunoconjugates ( 0.2 to $95 \mathrm{nmol} / \mathrm{L}$ in $100 \mu \mathrm{L}$ of PBS) for $4 \mathrm{~h}$ at $4^{\circ} \mathrm{C}$ with 200,000 cells. Non-specific binding was determined in a similar assay but in the presence of a 50-fold mole excess of nimotuzumab (relative to the highest concentration of the radio-immunoconjugates, i.e. $4,500 \mathrm{nmol} / \mathrm{L})$. A non-linear regression analysis with One Site - Total and Nonspecific binding equation was used to determine the $\mathrm{K}_{\mathrm{D}}$ and the binding potential (Bmax) from which the number of receptors per cell was determined (GraphPad Prism V5.02, 2008). Immunoreactivity fractions of ${ }^{89} \mathrm{Zr}$-DFO-nimotuzumab were determined as described by Lindmo et al. [22] using DLD-1 cells.

\section{Pharmacokinetics and dosimetry estimation}

Female athymic CD-1 mice $(\mathrm{N}=4)$ were injected intravenously via the tail vein with $6.5 \pm 0.0 .1$ (specific activity $0.38 \mathrm{MBq} / \mu \mathrm{g}){ }^{89} \mathrm{Zr}-\mathrm{DFO}$-nimotuzumab in PBS. Blood samples were collected from the saphenous vein at different time points $(0.08-144 \mathrm{~h})$ in a heparinized capillary tube and the blood radioactivity was expressed as the percentage of injected dose $/ \mathrm{mL}(\% \mathrm{ID} / \mathrm{mL})$. Pharmacokinetic parameters, including the distribution and elimination half-lives $\left(t_{1 / 2} \alpha\right.$ and $\left.t_{1 / 2} \beta\right)$, volume of distribution at steady-state (Vss), and clearance (CL) were estimated by fitting the blood radioactivity versus time curve to a two-compartment model with i.v. bolus input.

Human radiation dosimetry estimates were calculated from animal biodistribution data obtained by the standard method of organ dissection. Animal biodistribution data was obtained using Balb/c (50 males, 50 females) mice injected with $3.2 \pm 0.2 \mathrm{MBq}$ of ${ }^{89} \mathrm{Zr}$-DFO-nimotuzumab divided in 10 groups of 10 animals ( 5 females/5 males). Animals were euthanized and organs were harvested in groups of five at the following time points: $1,4,12,24,48,72,96,120,144$, and 168 hours post injection (hp.i). The following organs were harvested, weighed, and counted for radio-activity in a gamma counter: blood, lungs, liver, spleen, kidneys, bladder, muscle, heart, brain, bones, red marrow, testes, adrenals, pancreas, uterus, ovaries, stomach, small intestine, and large intestine. Radioactivity in organs was expressed as $\%$ injected activity ( $\%$ IA) or $\%$ IA per gram ( $\%$ IA $/ g)$. For each organ or tissue, the effective data (non-decay-corrected $\%$ IA/g) were plotted against sampling time, and used to obtain an estimate of the microcurie-hours per microcurie administered, represented by the area under the timeactivity function integrated to infinity (complete decay) of the ${ }^{89} \mathrm{Zr}$. Absorbed doses in units of cGy (centigray) per milicurie (cGy/mCi) Zr-89 administered were calculated. Mouse organ doses were extrapolated hypothetically to the human and recalculated by recalculating the residence times for the human model from the mouse model using OLINDA1.1 [17, 23]. 


\section{Single dose toxicity study of ${ }^{89} \mathrm{Zr}$-DFO- nimotuzumab in mice}

Acute (Day-2: considering Day 1 as the day of injection) and delayed (Day-14) toxicity studies was carried out in male and female ( $\mathrm{n}=5$ males +5 females), six week old Balb/C mice (Charles Rivers). Mice were monitored for weight and behavior changes. The vehicle for injection was PBS as it was used in the final preparation of ${ }^{89} \mathrm{Zr}$-DFO-nimotuzumab. $74 \mathrm{MBq}(10 \mathrm{mg})$ of immunoconjugates was proposed maximum dosage for human use. 100 mice were divided in to five groups: 20 mice were used for base line study, 40 mice (control group) were treated with vehicle (PBS). 20 of these were sacrificed as a control of the acute toxicity study on Day- 2 and 20 mice were sacrificed as a control of the delayed toxicity study on Day-14. 40 mice (treatment group) were injected with $3.2 \mathrm{MBq}, 0.065 \mathrm{mg}$ (which corresponds to 168 -fold excess radioactivity dose and 25 -fold excess mass dose of ${ }^{89} \mathrm{Zr}$-nimotuzumab that is projected for human studies on a weight/weight basis). 20 of these were sacrificed on Day-2 for the acute toxicity study and 20 of them were sacrificed for delayed toxicity study on Day-14.

All animals in this study were observed regularly for signs of mortality, morbidity, injury, and availability of food and water. Individual body weights were determined and recorded during the quarantine period and (every other day) experimental period. Blood was collected via cardiac puncture for hematology and clinical chemistry. A complete whole blood and plasma analyses were done. Mice were sacrificed on, day 2, or day 14 and organs of interest (kidneys, spleen, liver, bone, heart, lungs, brain, and testes/uterus) were harvested and stored in $10 \%$ formaldehyde solution. They were further processed and examined for histopathological analyses.

\section{Biodistribution and microPET imaging in tumor bearing mice}

Biodistribution studies of ${ }^{89} \mathrm{Zr}$-DFO-nimotuzumab was performed in mice bearing EGFR-positive MDAMB-468, DLD-1, and EGFR-negative MDA-MB-435 xenografts. Female CD-1 nude mice (4 to 6 weeks old) were obtained from Charles River and inoculated subcutaneously with $10^{6}$ cells on the right flank. Mice ( $\mathrm{n}=4$ per time point) were injected with $10 \pm 1 \mathrm{MBq} 10$ $\mu \mathrm{g}{ }^{89} \mathrm{Zr}$-DFO-nimotuzumab injection via tail vein and sacrificed at 4, 24, 72, 120,144, or $168 \mathrm{~h}$ post injection. Blood and major organs were collected and weighed. The radioactivity in blood and organs was counted using an automated $\gamma$-counter and expressed as a percentage of injected activity per gram of organ weight (\% IA/g).

Tumor bearing mice were injected via a tail vein with 10-12 MBq $10-12 \mu \mathrm{g}$ of ${ }^{80} \mathrm{Zr}$-DFO-nimotuzumab. PET/CT images were acquired at 4, 24, 48, 72, 96, 120, 144, and 168 hp.i using the Vector4CT scanner (MILabs B.V., Utrecht,)
PET scans were acquired in a list-mode data format with an high-energy ultra-high resolution (HE-UHR-1.0 mm) mouse/rat pinhole collimator. Corresponding CT scans were acquired with a tube setting of $50 \mathrm{kV}$ and $480 \mu \mathrm{A}$. Images were reconstructed using a pixel-based ordersubset expectation maximization (POS-EM) algorithm that included resolution, recovery and compensation for distance-dependent pinhole sensitivity and were registered on CT and quantified using PMOD 3.8 software (PMOD, Switzerland). Tracer uptake was expressed as percentage injected activity (\% IA) per cc of tissue volume (\% IA/cc). All quantification data was reported as mean \pm standard deviation within one animal study group.

\section{Statistical analysis}

All data was expressed as the mean \pm Stdev of at least 3 independent experiments. Statistical significance between groups was assessed using a two-tailed Student's $t$-test or analysis of variance (ANOVA) with Bonferoni post hoc test. All graphs were prepared and analysed using GraphPad Prism (version 5; GraphPad, La Jolla, CA).

\section{Abbreviations}

Positron Emission Tomography (PET), Epidermal Growth Factor Receptor I (EGFR), Desferoxamine (DFO), miliSievert (mSv), mega Becquerel (MBq), microCurie (mCi), Zirconium (Zr), Roentgen Equivalent Man (rem), Immunohistochemistry (IHC), Fluorescence In Situ Hybridization (FISH), Percent Injected Activity (\%IA) per gram (\%IA/g), Quantitative Polymerase Chain Reaction (qPCR), High-Energy Ultra-High Resolution (HE-UHR), Good Manufacturing Practice (GMP), centiGray (cGy), Size Exclusion Column (SEC), hour post injection (h.p.i).

\section{Author contributions}

R.C. and V.R.S. designed and performed experiments (labeling, stability studies, biodistribution), E.A. designed and performed experiments (microPET imaging experiments), analyzed data. W.B. performed in vitro characterization (bioanalyzer, flow cytometry) of the antibody conjugates. W.H. performed binding kinetic studies (octet). D.F. performed dosimetry estimation using biosdistribution data. J.F.D. performed pathological examination of the slides. K.B. designed and supervised experiments, analyzed data, and reviewed and edited the manuscript. R.G. supervised the study, reviewed, and edited the manuscript. H.F. supervised the study, wrote and edited the manuscript.

\section{ACKNOWLEDGMENTS}

Nimotuzumab was provided by the Center for Molecular Immunology (Havana, Cuba) under the terms of 
a research collaboration. The authors wish to acknowledge the technical contributions of Dr. Hartimath Siddanna and Mrs. Carolina Gonzalez.

\section{CONFLICTS OF INTEREST}

Authors declare no conflicts of interest.

\section{FUNDING}

This study was funded by grants from the Canadian Breast Cancer Research (No. 300030), Sylvia Fedoruk Canadian Centre for Nuclear Innovation (J2016-0036), Saskatchewan Health Research Foundation (No. 3554) and Western Economic Diversification (\#12939).

\section{REFERENCES}

1. Chung CH, Ely K, McGavran L, Varella-Garcia M, Parker J, Parker N, Jarrett C, Carter J, Murphy BA, Netterville J, Burkey BB, Sinard R, Cmelak A, et al. Increased epidermal growth factor receptor gene copy number is associated with poor prognosis in head and neck squamous cell carcinomas. J Clin Oncol. 2006; 24:4170-6.

2. Alshenawy HA. Immunohistochemical expression of epidermal growth factor receptor, E-cadherin, and matrix metalloproteinase-9 in ovarian epithelial cancer and relation to patient deaths. Ann Diagn Pathol. 2010; 14:387-95.

3. Cunningham D, Humblet Y, Siena S, Khayat D, Bleiberg H, Santoro A, Bets D, Mueser M, Harstrick A, Verslype C, Chau I, Van Cutsem E. Cetuximab monotherapy and cetuximab plus irinotecan in irinotecan-refractory metastatic colorectal cancer. N Engl J Med. 2004; 351:337-45.

4. Giltnane JM, Ryden L, Cregger M, Bendahl PO, Jirstrom K, Rimm DL. Quantitative measurement of epidermal growth factor receptor is a negative predictive factor for tamoxifen response in hormone receptor positive premenopausal breast cancer. J Clin Oncol. 2007; 25:3007-14.

5. Bellone S, Frera G, Landolfi G, Romani C, Bandiera E, Tognon G, Roman JJ, Burnett AF, Pecorelli S, Santin AD. Overexpression of epidermal growth factor type-1 receptor (EGF-r1) in cervical cancer: implications for cetuximabmediated therapy in recurrent/metastatic disease. Gynecol Oncol. 2007; 106:513-20.

6. Garrido G, Tikhomirov IA, Rabasa A, Yang E, Gracia E, Iznaga N, Fernández LE, Crombet T, Kerbel RS, Pérez R. Bivalent binding by intermediate affinity of nimotuzumab: a contribution to explain antibody clinical profile. Cancer Biol Ther. 2011; 11:373-82.

7. Spano JP, Lagorce C, Atlan D, Milano G, Domont J, Benamouzig R, Attar A, Benichou J, Martin A, Morere JF, Raphael M, Penault-Llorca F, Breau JL, et al. Impact of EGFR expression on colorectal cancer patient prognosis and survival. Ann Oncol. 2005; 16:102-8.
8. Italiano A, Follana P, Caroli FX, Badetti JL, Benchimol D, Garnier G, Gugenheim J, Haudebourg J, Keslair F, Lesbats G, Lledo G, Roussel JF, Pedeutour F, et al. Cetuximab shows activity in colorectal cancer patients with tumors for which fish analysis does not detect an increase in EGFR gene copy number. Ann Surg Oncol. 2008; 15:649-54.

9. Jakobsen JN, Sorensen JB. Intratumor heterogeneity and chemotherapy-induced changes in EGFR status in nonsmall cell lung cancer. Cancer Chemother Pharmacol. 2012; 69:289-99.

10. Fasih A, Fonge H, Cai Z, Leyton JV, Tikhomirov I, Done SJ, Reilly RM. In-111-bn-DTPA-nimotuzumab with/without modification with nuclear translocation sequence (NLS) peptides: an auger electron-emitting radioimmunotherapeutic agent for EGFR-positive and trastuzumab (herceptin)-resistant breast cancer. Breast Cancer Res Treat. 2012; 135:189-200.

11. van Loon J, Even AJ, Aerts HJ, Ollers M, Hoebers F, van Elmpt W, Dubois L, Dingemans AC, Lalisang RI, Kempers P, Brans B, Winnepenninckx V, Speel EJ, et al. PET imaging of zirconium-89 labelled cetuximab: a phase i trial in patients with head and neck and lung cancer. Radiother Oncol. 2017; 122:267-73.

12. Garousi J, Andersson KG, Mitran B, Pichl ML, Stahl S, Orlova A, Lofblom J, Tolmachev V. PET imaging of epidermal growth factor receptor expression in tumours using ${ }^{89}$ Zr-labelled ZEGFR:2377 affibody molecules. Int J Oncol. 2016; 48:1325-32.

13. Chang AJ, De Silva RA, Lapi SE. Development and characterization of 89zr-labeled panitumumab for immunopositron emission tomographic imaging of the epidermal growth factor receptor. Mol Imaging. 2013; 12:17-27.

14. Menke-van der Houven van Oordt CW, Gootjes EC, Huisman MC, Vugts DJ, Roth C, Luik AM, Mulder ER, Schuit RC, Boellaard R, Hoekstra OS, van Dongen GA, Verheul HM. ${ }^{89} \mathrm{zr}$-cetuximab PET imaging in patients with advanced colorectal cancer. Oncotarget. 2015; 6:30384-93. https://doi.org/10.18632/oncotarget.4672.

15. Vallis KA, Reilly RM, Chen P, Oza A, Hendler A, Cameron R, Hershkop M, Iznaga-Escobar N, Ramos-Suzarte M, Keane P. A phase i study of tc-99m-hr3 (diaCIM (R)), a humanized immunoconjugate directed towards the epidermal growth factor receptor. Nucl Med Commun. 2002; 23:1155-64.

16. Bhattacharyya S, Kurdziel K, Wei L, Riffle L, Kaur G, Hill GC, Jacobs PM, Tatum JL, Doroshow JH, Kalen JD. Zirconium-89 labeled panitumumab: a potential immunoPET probe for HER1-expressing carcinomas. Nucl Med Biol. 2013; 40:451-7.

17. Laforest R, Lapi SE, Oyama R, Bose R, Tabchy A, Marquez-Nostra BV, Burkemper J, Wright BD, Frye J, Frye S, Siegel BA, Dehdashti F. [Zr-89]trastuzumab: evaluation of radiation dosimetry, safety, and optimal imaging parameters in women with HER2-positive breast cancer. Mol Imaging Biol. 2016; 18:952-9. 
18. Marti-Climent JM, Prieto E, Moran V, Sancho L, Rodriguez-Fraile M, Arbizu J, Garcia-Velloso MJ, Richter JA. Effective dose estimation for oncological and neurological PET/CT procedures. EJNMMI Res. 2017; $7: 37$.

19. Even AJG, Hamming-Vrieze O, van Elmpt W, Winnepenninckx VJL, Heukelom J, Tesselaar MET, Vogel WV, Hoeben A, Zegers CML, Vugts DJ, van Dongen GAMS, Bartelink H, Mottaghy FM, et al. Quantitative assessment of zirconium-89 labeled cetuximab using PET/ CT imaging in patients with advanced head and neck cancer: a theragnostic approach. Oncotarget. 2017; 8:3870 80. https://doi.org/10.18632/oncotarget.13910.

20. Dijkers EC, Oude Munnink TH, Kosterink JG, Brouwers AH, Jager PL, de Jong JR, van Dongen GA, Schroder CP, Lub-de Hooge MN, de Vries EG. Biodistribution of 89zr-trastuzumab and PET imaging of HER2-positive lesions in patients with metastatic breast cancer. Clin Pharmacol Ther. 2010; 87:586-92.

21. Deri MA, Zeglis BM, Francesconi LC, Lewis JS. PET imaging with (8)(9)Zr: from radiochemistry to the clinic. Nucl Med Biol. 2013; 40:3-14.

22. Lindmo T, Boven E, Cuttitta F, Fedorko J, Bunn PA. Determination of the immunoreactive fraction of radiolabeled monoclonal-antibodies by linear extrapolation to binding at infinite antigen excess. J Immunol Methods. 1984; 72:77-89.

23. Kirschner AS, Ice RD, Beierwaltes WH. Radiationdosimetry of I-131-19-iodocholesterol - pitfalls of using tissue concentration data - reply. J Nucl Med. 1975; 16:248-9. 